\title{
VARIAÇÕES FISIOLÓGICAS DA PRESSÃO DO LIQUIDO CEFALORRAQUEANO NA CISTERNA MAGNA
}

\author{
A. Spina-França *
}

A pressão normal do líquido cefalorraqueano (LCR) ao nivel do fundo de saco lombar varia entre 7 e $18 \mathrm{~cm}$ de água, quando o paciente se encontra em decúbito lateral horizontal. São considerados aumentados os valôres maiores do que 20. Quando a pressão do LCR está entre 18 e $20 \mathrm{~cm}$ de água, é considerada dado duvidoso no sentido de caracterizar a existência de hipertensão liquórica. A essas conclusões chegaram Merritt e Fremont-Smith ${ }^{5}$ pelo estudo da pressão inicial do LCR de 1.033 pacientes. Em estudos posteriores êsses limites de normalidade são adotados ${ }^{4}$ ou servem de referência ${ }^{1}$.

$\mathrm{Na}$ posição horizontal a pressão do LCR ao nivel da cisterna magna (punção suboccipital) costuma ser discretamente menor do que na região lombar (2 a $3 \mathrm{~cm}$ de água, segundo Lange ${ }^{2}$ ). Considerando a existência de tais diferenças, Longo, Dourado e Reis ${ }^{3}$ analisaram os valôres normais da pressão do nível da cisterna magna. Para um grupo de 96 casos encontraram como valôres máximo e mínimo, respectivamente, 19,5 e $5,5 \mathrm{~cm}$ de água.

No estudo presente é feita reavaliação do problema dos limites das variações fisiológicas da pressão inicial do LCR, ao mesmo tempo em que é analisada a influência do sexo, da idade e da côr dos pacientes e são caracterizadas as diferenças de pressão entre a cisterna magna e o fundo de saco lombar.

\section{MATERIAL E MÉTODOS}

As variaçōes fisiológicas da pressão inicial do LCR ao nivel da cisterna magna foram analisadas através dos dados referentes a 5 grupos de pacientes. Estes eram portadores de doenças do sistema nervoso mas não apresentavam sintomatologia de hipertensão ou de hipotensāo intracraniana. Os casos foram selecionados entre aquêles submetidos sucessivamente a exames do LCR no ambulatório da Clínica Neurológica da Faculdade de Medicina da Universidade de São Paulo.

Em todos os casos foi estudada a pressão inicial, isto é, a pressão antes da retirada de amostra de LCR. A determinação da pressão foi feita com o paciente em

Trabalho da Clínica Neurológica da Fac. Med. da Univ. de São Paulo (Prof. A. Tolosa): * Assistente-doutor. 
decúbito lateral horizontal e os valôres computados se referem a pacientes que não apresentavam distúrbios emocionaís ligados ao ato de punção, isto é, que se achavam calmos. Para as determinações foi utilizado manômetro aneróide graduado em $\mathrm{cm}$ de água (manômetro de Claude).

Nos 4 primeiros grupos de casos a pressão foi medida ao nivel da cisterna magna (punção suboccipital). O primeiro grupo de casos compreende 1.500 pacientes adultos, cuja pressão do LCR se distribuia entre 0 e $25 \mathrm{~cm}$ de água; os dados dêsse grupo serviram para o cálculo das estimativas da normalidade. Em relação aos parâmetros dêsse grupo, foram selecionados os casos dos três grupos seguintes, destinados ao estudo da influência do sexo, da raça e da idade. A influência do sexo é estudada no segundo grupo de casos, que compreende 100 pacientes adultos do sexo masculino e 100, do feminino. A influência da côr é analisada no terceiro grupo, que compreende 100 pacientes adultos de côr branca, 100 de côr preta, 100 pardos e 100 de côr amarela. A influência do fator etário foi estudada pela análise dos valôres de pressão dos pacientes do quarto grupo (605 casos). Estes foram distribuídos segundo grupos etários de 10 anos, entre as idades limites de 1 e 70 anos.

o quinto grupo de casos compreende as pressōes encontradas no fundo de saco lombar (punção lombar) de 276 pacientes adultos. A seleção dêsses casos fol feita segundo os mesmos critérios adotados em relação ao primeiro grupo. As diferenças de pressão ligadas ao local de punção foram estudadas pela análise comparativa dos dados do primeiro e do quinto grupo.

\section{RESULTADOS}

Os resultados referentes aos 1.500 pacientes reunidos no primeiro grupo de casos são apresentados no quadro 1. Constam do quadro 2 aquêles referentes aos casos do segundo e do terceiro grupo (influência do sexo e da côr, respectivamente). No quadro 3 são apresentados os dados referentes ao quarto grupo de casos (análise do fator etário) e no quadro 4, aquêles referentes ao quinto grupo (pressão na região lombar).

\begin{tabular}{|c|c|c|c|c|c|c|c|}
\hline \multicolumn{2}{|c|}{ Pressão (cm de água) } & \multirow{2}{*}{$\begin{array}{r}N^{\circ} \\
\\
\end{array}$} & \multirow{2}{*}{$\underbrace{d e \text { casos }}_{0}$} & \multicolumn{2}{|c|}{ Pressão (cm de água) } & \multirow{2}{*}{ No } & \multirow{2}{*}{$\underbrace{d e \text { casos }}_{180}$} \\
\hline $0-1$ & $\ldots \ldots \ldots \ldots \ldots \ldots$ & & & $14-15$ & $\ldots \ldots \ldots \ldots \ldots \ldots$ & & \\
\hline $2-3$ & $\ldots \ldots \ldots \ldots \ldots$ & & 16 & $16-17$ & $\ldots \ldots \ldots \ldots \ldots$ & & 95 \\
\hline 4- 5 & $\ldots \ldots \ldots \ldots \ldots \ldots$ & & 39 & $18-19$ & $\ldots \ldots \ldots \ldots \ldots \ldots$ & & 63 \\
\hline 6- 7 & $\ldots \ldots \ldots \ldots$ & & 110 & $20-21$ & $\ldots \ldots \ldots \ldots \ldots \ldots$ & & 34 \\
\hline $8-9$ & $\ldots \ldots \ldots \ldots$ & & 236 & $22-23$ & $\ldots \ldots \ldots \ldots \ldots$ & & 21 \\
\hline 10-11 & $\ldots \cdots \cdots \cdots \cdots$ & & 341 & $24-25$ & $\ldots \ldots \ldots \ldots \ldots$ & & 31 \\
\hline 12-13 & $\ldots$ & & 334 & & & & \\
\hline
\end{tabular}

Média (cm de água) 11,9; desvio padrão 4,0.

Quadro 1 - Distribuição segundo a freqüência e estimativas referentes à pressão inicial do LCR ao nivel da cisterna magna (1.500 casos). 


\begin{tabular}{|c|c|c|c|c|c|c|}
\hline \multirow{2}{*}{ Pressão (cm de água) } & \multicolumn{2}{|c|}{ Sexo } & \multicolumn{4}{|c|}{$C$ ôr } \\
\hline & masc. & fem. & branca & preta & parda & amarela \\
\hline 4- 5 & 1 & 1 & 4 & 6 & 3 & 11 \\
\hline 6- $7 \quad \ldots$ & 1 & 4 & 9 & 13 & $\mathbf{5}$ & 12 \\
\hline $8-9 \quad \ldots$ & 17 & 21 & 11 & 11 & 11 & 13 \\
\hline $10-11 \quad \ldots$ & 29 & 21 & 21 & 34 & 24 & 26 \\
\hline $12-13 \quad \ldots \ldots \ldots$ & 29 & 26 & 23 & 13 & 20 & 17 \\
\hline 14-15 $\ldots$ & 8 & 8 & 14 & 13 & 19 & 10 \\
\hline $16-17 \quad \ldots$ & 10 & 5 & 4 & 2 & 12 & 4 \\
\hline $18-19$ & 4 & 9 & 8 & 5 & 5 & $\mathbf{3}$ \\
\hline$\ldots \ldots \ldots$ & 1 & 5 & 6 & 3 & 1 & 4 \\
\hline No de casos .. & 100 & 100 & 100 & 100 & 100 & 100 \\
\hline Média $(\mathrm{cm}$ de água) $\ldots$. & 11,9 & 12,2 & 12,0 & 10,8 & 12,1 & 10,4 \\
\hline Desvio padrão $\ldots \ldots \ldots$. & 3,0 & $\mathbf{3 , 5}$ & 4,0 & 3,7 & 3,4 & 3,9 \\
\hline
\end{tabular}

Quadro 2 - Distribuição dos casos dos segundo (sexo) e terceiro (côr) grupos, de acôrdo com a pressão inicial do LCR na cisterna magna.

\begin{tabular}{|c|c|c|c|c|c|c|c|}
\hline \multirow{2}{*}{$\begin{array}{c}\text { Pressão } \\
\text { (cm de água) }\end{array}$} & \multicolumn{7}{|c|}{ Idade (em anos) } \\
\hline & $1-10$ & $11-20$ & $21-30$ & $31-40$ & $41-50$ & $51-60$ & $61-70$ \\
\hline $4-5 \ldots \ldots \ldots$ & 0 & 0 & 3 & 2 & 5 & 4 & 2 \\
\hline $6-7 \ldots \ldots \ldots$ & 1 & 4 & 5 & 8 & 1 & 3 & 4 \\
\hline 8- $9 \ldots$ & 1 & 11 & 23 & 15 & 11 & 5 & 1 \\
\hline $10-11 \ldots \ldots \ldots$ & 10 & 24 & 34 & 30 & 22 & $\mathbf{5}$ & 4 \\
\hline $12-13$ & 8 & 36 & 44 & 29 & 16 & 5 & 5 \\
\hline $14-15 \ldots \ldots \ldots$ & 6 & 24 & 36 & 28 & 24 & 8 & 2 \\
\hline $16-17 \ldots \ldots \ldots$ & 4 & 9 & 9 & 9 & 3 & 6 & 0 \\
\hline $18-19 \ldots \ldots \ldots$ & 1 & 7 & 6 & 10 & 8 & 3 & 0 \\
\hline 20 & 2 & 5 & 3 & 6 & 4 & 0 & 1 \\
\hline Ne de casos $\ldots$. & 33 & 120 & 163 & 137 & 94 & 39 & 19 \\
\hline Pressão média & 12,9 & 12,7 & 12,0 & 12,5 & 12,3 & 11,8 & 10,2 \\
\hline
\end{tabular}

Quadro 3 - Distribuicão dos 605 casos (quarto grupo) segundo a idade e a pressāo inicial do LCR na cisterna magna. 


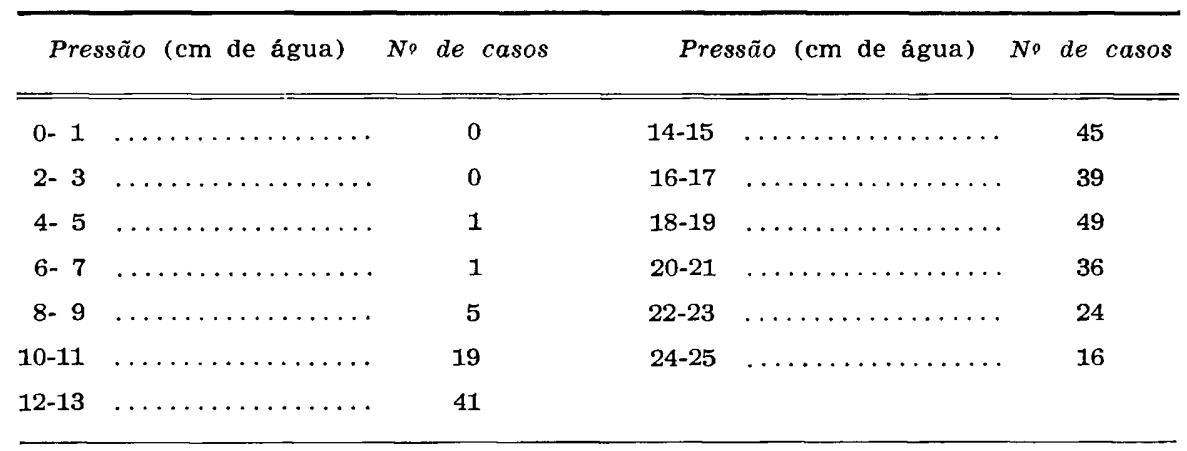

Média (cm de água) 16,7; desvio padrão 4,1.

Quadro 4 - Distribuicão dos 276 casos do quinto grupo conforme a pressão do LCR na região lombar.

\section{COMENTARIOS}

Os dados analisados permitem caracterizar a pressão normal e as variações fisiológicas da pressão do LCR ao nivel da cisterna magna, quando ela é medida com o paciente calmo e em decúbito lateral horizontal. Igualmente, permitem estudar o significado das variações ligadas ao sexo, à côr e à idade dos pacientes. As diferenças entre a pressão normal média ao nivel da cisterna magna e do fundo de saco lombar também podem ser avaliadas por êste estudo.

Pressão normal e limites das variações fisiológicas - Pela análise dos dados referentes aos 1.500 casos do primeiro grụ̣o o valor médio da pressão inicial do LCR ao nivel da cisterna magna é representado por $11,9 \mathrm{~cm}$ de água; os limites das variações fisiológicas $(t=5 \%)$ são representados pelas pressões de 4,1 e $19,7 \mathrm{~cm}$ de água. Esses limites permitem estabelecer que os valôres de pressão iguais ou menores do que $4 \mathrm{~cm}$ de água são representativos de existência de hipotensão do LCR na cisterna magna. Os valôres de pressão iguais ou maiores do que $20 \mathrm{~cm}$ de água caracterizam, por sua vez, a existência de hipertensão.

Variações ligadas ao sexo - As estimativas encontradas para os pacientes do sexo masculino e do sexo feminino mostram diferenças não significativas entre si $(t=0,65)$. Esse fato vem comprovar que a pressão inicial do LCR não sofre variações relacionadas ao sexo.

Variações ligadas $\grave{a}$ côr - Os pacientes brancos representam a maior amostra de nossa população, tanto que era esta a côr da maioria dos pacientes do primeiro grupo. Em relação às estimativas dêstes não se mostra significativa a diferença achada para a pressão do grupo de pacientes de côr branca estudado em separado no terceiro grupo de casos $(t=0,25)$. 
Tomando êste último grupo de brancos como têrmo de comparação, as diferenças achadas em relação aos pacientes pardos se mostra significante $(t=0,24)$. As diferenças em relação aos pacientes prêtos e amarelos, porém foram significativas ( $t=2,18$ para prêtos e $t=2,86$ para amarelos). Êsses dados vêm mostrar que os valôres de pressão entre os prêtos e amarelos podem apresentar-se discretamente inferiores que aquêles encontrados na raça branca. A diferença encontrada não apresenta significado do ponto de vista clínico.

Variações ligadas à idade — Os valôres médios representativos da pressão do LCR para os 605 casos em que esta foi distribuída segundo grupos etários são apresentados no quadro 3. A média mais alta foi encontrada para o primeiro grupo etário (pacientes com idade entre 1 e 10 anos) e a menor, para o último (pacientes com idade entre 61 e 70 anos). Entre êsses extremos os valôres médios decaíram progressivamente. As diferenças entre as médias não apresentam maior significado do ponto de vista clínico por serem muito pequenas. $\dot{E}$ de notar que a tendência à diminuição da pressão média se acentuou entre o penúltimo e o último grupo etário. Êste dado é sugestivo de a pressão ser menor na senescência. Este fato não pode ser afirmado em relação aos dados apresentados pelo fato de ser pequeno o número de pacientes dêste último grupo.

Diferenças entre a pressão ao nível da cisterna magna e do fundo de saco lombar - A média achada para os 1.500 casos em que a pressão foi medida na cisterna magna foi de $11,9 \mathrm{~cm}$ de água; para os 276 casos em que foi medida na região lombar, de 16,7. A diferença entre essas duas médias é altamente significativa $(t=20,0)$, comprovando que a pressão do LCR costuma ser maior ao nivel do fundo de saco lombar (paciente em decúbito lateral).

\section{RESUMO E CONCLUSÕES}

É feito estudo de revisão das variações fisiológicas da pressão do LCR ao nível da cisterna magna (punção suboccipital).

Em relação à série de 1.500 pacientes com afecções do sistema nervoso e sem sintomatologia de hipotensão ou de hipertensão intracraniana, a pressão média normal do LCR ao nível da cisterna magna foi de $11,9 \mathrm{~cm}$ de água (paciente calmo, decúbito lateral horizontal). As variações fisiológicas de pressão, segundo os achados, têm como limites os valôres de 4,1 e $19,7 \mathrm{~cm}$ de água. Êsses valôres permitem caracterizar, para a cisterna magna, a existência de hipotensão do LCR quando a pressão fôr menor ou igual a $4 \mathrm{~cm}$ de água; a hipertensão é caracterizada por valôres iguais ou maiores do que $20 \mathrm{~cm}$ de água.

A pressão do LCR ao nível da cisterna magna é menor do que no fundo de saco lombar. Para série de 276 pacientes o valor médio achado para êste último nivel foi de $16,7 \mathrm{~cm}$ de água (decúbito lateral horizontal). A diferença entre as médias foi altamente significativa. 
Não foram verificadas modificações da pressão do LCR ao nivel da cisterna magna que pudessem ser relacionadas ao sexo. Sem que representassem dados de interêsse para a prática, foram verificadas variações ligadas à côr e à idade dos pacientes. Em média a pressão do LCR se mostrou menor entre pacientes prêtos e amarelos que entre os brancos e os mulatos. Um declínio do valor médio da pressão foi verificado à medida em que aumentava a idade dos pacientes. Este declínio mostrou-se maior para o grupo de pacientes com idade acima de 60 anos.

\section{SUMMARY}

\section{Physiological variations of the cerebrospinal fluid pressure in the cisterna magna.}

Data concerning to the normal pressure of the CSF at the level of the cisterna magna were reviewed. The values found for 1,500 patients with diseases of the nervous system but without symptomatology of intracranial hypotension or hypertension were analysed. The determination of the pressure was made with the patients relaxed, in horizontal and lateral decubitus. The mean value found was that of $11.9 \mathrm{~cm}$ of water and the limits for the physiological variations were 4.1 and $19.7 \mathrm{~cm}$ of water. Thus, hypotension is characterized when the pressure is $4 \mathrm{~cm}$ of water or lower. Hypertension is to be referred when $20 \mathrm{~cm}$ of water or more represents the CSF pressure in the cisterna magna.

The CSF pressure is lower in the cisterna magna than in the lumbar region. In a group of 276 patients the mean value found for the later was $16.7 \mathrm{~cm}$ of water (lateral horizontal decubitus). The difference between the values showed to be significant.

There are not differences related to the sex in the CSF pressure at the cisterna magna. There are significative differences related to the colour of the patients. The values were found to be lower among black and yellow patients. The mean pressure tends to be lower with the age. This tendency was found to increase over 60 years old. The differences related to the colour and to the age of the patients which were found, have not clinical significance.

\section{REFERENCIAS}

1. HEMmer, R. - Der Liquordruck. G. Thieme Verlag, Stuttgart, 1960. 2. LANGE, O. - O Liquido Céfalo-Raqueano em Clinica. Cia. Melhoramentos de Sāo Paulo, 1937 3. LONGO, P. W.; DOURADO, J. V.; REIS, J. B. - Pressão do líquido cefalorraqueano. Conceito de normalidade. Rev. Paulista Med. 36:45-48 (janeiro) 1950. 4. LUPS, S.; HAAN, A. M. F. H. - The Cerebrospinal Fluid. Elsevier Publ. Co., Amsterdam, 1954. 5. MERRITT, H. H.; FREMONT-SMITH, F, - The Cerebrospinal Fluid. W. B. Saunders Co., Philadelphia, 1937.

Clinica Neurológica - Faculdade de Medicina da USP - Caixa Postal 3461 São Paulo, SP - Brasil. 